\title{
Tarifrevision: TARMED CONSENSUS (TARCO) ist on track
}

\section{Urs Stoffel}

Dr. med., Mitglied des FMH-Zentralvorstandes, Departementsverantwortlicher Ambulante Versorgung und Tarife

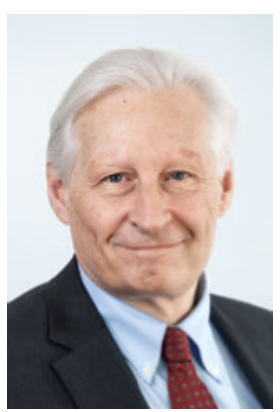

TARCO ist der einzige Ausweg aus dem Amtstarif, der ab 1. Januar 2018 Realität wird. Googelt man den Begriff "on track», so findet man Interpretationen wie: "auf der Spur» oder "wieder auf dem richtigen Weg» sein. Das Projekt TARCO ist also auf dem richtigen Weg und auf Zielkurs!

An seiner Sitzung vom 29. November 2017 hat das COCKPIT über 90 Prozent der Nomenklatur der Tarifstruktur verabschiedet. Einige wenige Differenzen müssen in den nächsten Tagen noch ausgeräumt und letzte übergreifende Kapitel finalisiert werden.

Das Steuerungsorgan COCKPIT setzt sich aus Vertretern aller Dachverbände und Basisorganisationen der FMH zusammen und wurde von der Delegiertenversammlung der FMH als oberstes Entscheidungsorgan des Projekts TARCO eingesetzt.

Das nächste grosse Etappenziel auf dem Weg zu einer gemeinsamen Einreichung der Tarifstruktur TARCO ist die Verhandlung mit den Tarifpartnern; erste Verhandlungen in ausgewählten Bereichen haben bereits begonnen oder werden zurzeit aufgegleist.

\section{TARCO ist der einzige Ausweg aus dem Amtstarif, der ab 1. Januar 2018 Realität wird.}

Seit der Freigabe der Delegiertenversammlung der FMH für das Projekt TARCO im Januar 2017 arbeiten über 150 Tarifdelegierte und Experten in über $40 \mathrm{Ar}$ beitsgruppen an der Revision. Ausgangspunkt von TARCO war die Tarifversion ats-tms 1.0 (TARVISION), welche im Juni 2016 von den Mitgliedern der FMH in einer Urabstimmung abgelehnt wurde. Sämtliche Kapitel aus der Vorgängerversion wurden im Konsens mit allen Fachgesellschaften nochmals überarbeitet und gemäss den Tarifierungsgrundsätzen TARCO homogenisiert.

Jedes Kapitel wurde jeweils in einer der vier Vernehmlassungsphasen allen Fachgesellschaften für eine qualifizierte Rückmeldung zur Verfügung gestellt. An- schliessend wurden die Feedbacks gesichtet und wo sinnvoll eingearbeitet.

Gegenüber der noch gültigen TARMED-Version 1.08_ BR mit 4519 Tarifpositionen umfasst die ambulante Tarifstruktur TARCO noch rund 2700 Tarifpositionen und damit 40 Prozent weniger Tarifpositionen. Sofort hören wir den Vorwurf: «Das sind doch immer noch viel zu viele Tarifpositionen, das muss noch einfacher werden.» Es gilt festzuhalten, dass der TARMED ein ambulanter Einzelleistungstarif ist, in welchem alle Pflichtleistungen gemäss der Krankenpflege-Leistungsverordnung KLV abgebildet werden müssen. Gleichzeitig mit dem Vorwurf, die Tarifstruktur TARMED sei

\section{Erste Verhandlungen mit den Tarifpart-} nern in ausgewählten Bereichen haben bereits begonnen.

viel zu umfangreich und komplex, hören wir auch den Vorwurf, der Arzttarif sei eine "Blackbox», niemand könne nachvollziehen, was überhaupt gemacht wurde. Damit stehen sich die Forderungen nach Transparenz und gleichzeitig nach weniger Komplexität diametral gegenüber. Hingegen wissen wir, dass das heute durch den TARMED generierte Kostenvolumen zu 97 Prozent durch nur 200 Tarifpositionen abgedeckt wird.

Auch der Ruf nach Pauschalen im ambulanten Tarif TARMED wird von der FMH sehr wohl gehört. Wir sind sehr gerne bereit, diese Pauschalen - wo möglich und sinnvoll - in den ambulanten Tarif TARMED einzubauen. Dabei werden Einzelleistungen zu Pauschalen gebündelt und so der Tarif vereinfacht. Letztlich ist aber die Basis jeder Pauschale ein sachgerechter und betriebswirtschaftlicher Einzelleistungstarif. Erst wenn bekannt ist, von welchen Leistungen man spricht, lassen sich diese auch transparent zu Pauschalen zusammenfassen.

Wir sind zuversichtlich, einen konsensfähigen, zukunftsweisenden und vor allem sachgerechten Tarif zu schaffen. Wir sind on track! 